\title{
Acylcarnitine profile analysis
}

\author{
Piero Rinaldo, $\mathrm{MD}, \mathrm{PhD}^{1}$, Tina M. Cowan, $P h D^{2}$, and Dietrich Matern, $M D^{1}$
}

Key Words: clinical genetic testing, technical standards and guidelines, acylcarnitine profile, inborn errors of metabolism, tandem mass spectrometry

\begin{abstract}
Disclaimer: These Technical Standards and Guidelines were developed primarily as an educational resource for clinical laboratory geneticists to help them provide quality clinical laboratory genetic services. Adherence to these standards and guidelines is voluntary and does not necessarily assure a successful medical outcome. These Standards and Guidelines should not be considered inclusive of all proper procedures and tests or exclusive of other procedures and tests that are reasonably directed to obtaining the same results. In determining the propriety of any specific procedure or test, the clinical laboratory geneticist should apply his or her own professional judgment to the specific circumstances presented by the individual patient or specimen. Clinical laboratory geneticists are encouraged to document in the patient's record the rationale for the use of a particular procedure or test, whether or not it is in conformance with these Standards and Guidelines. They also are advised to take notice of the date any particular standard or guidelines was adopted, and to consider other relevant medical and scientific information that becomes available after that date.

Genet Med 2008:10(2):151-156.
\end{abstract}

Acylcarnitine profile (ACP) analysis is performed for the biochemical screening of disorders of fatty acid oxidation (FAO) and organic acid metabolism. ${ }^{1,2}$ In some cases, ACP results are diagnostic for a specific disorder, while in other cases, further testing is needed to establish a precise diagnosis. ${ }^{1-3}$ The conditions revealed by acylcarnitine analysis have in common the accumulation of C2-C18 acyl-CoA species, which are substrates for one of several carnitine acyl-CoA transferases expressed in different intracellular compartments. The resulting acylcarnitine species are measured in the following clinical situations:

1. Evaluation of symptomatic patients

2. Evaluation of asymptomatic (at-risk) siblings of known patients

3. Newborn screening and follow-up testing

4. Prenatal diagnosis

5. Postmortem screening

Virtually all laboratories providing this analysis as a clinical service do so using tandem mass spectrometry (MS/MS) as the analytical platform. ${ }^{4-6}$ Less frequently employed methodologies include gas chromatography-mass spectrometry, highperformance liquid chromatography, and capillary electrophoresis. As is the case for all complex metabolic profiles, appropriate acylcarnitine analysis requires both analytical pro-

From the ${ }^{1}$ Biochemical Genetics Laboratory, Mayo Clinic College of Medicine, Rochester, Minnesota; and ${ }^{2}$ Department of Pathology, Stanford University Medical Center, Stanford, California.

American College of Medical Genetics, 9650 Rockville Pike Bethesda, MD 20814-3998.

Approved by the Board of Directors March 21, 2007.

Submitted for publication November 3, 2007.

Accepted for publication November 5, 2007.

DOI: 10.1097/GIM.0b013e3181614289 ficiency and in-depth interpretation of results with informative reporting.

\section{BACKGROUND}

\section{Clinical description of disease}

Inherited FAO disorders are inborn errors of metabolism (IEM) of relatively recent discovery. ${ }^{7-9}$ They may present at any age, from birth to adulthood, frequently leading to life-threatening episodes of metabolic decompensation after a period of inadequate caloric intake and intercurrent illness. Typical manifestations are hypoketotic hypoglycemia, liver disease, skeletal and cardiomyopathy, and sudden unexpected death.

Organic acidemias are a more heterogeneous group of IEM.10,11 They typically present with recurrent episodes of acute life-threatening illness, hypo- or hypertonia, failure to thrive, and developmental delay. Common acute manifestations include vomiting, lethargy, coma, and seizures.

With rare exceptions, the diagnosis of these conditions is almost exclusively a laboratory process, of which acylcarnitine analysis is a key component. A comprehensive metabolic evaluation typically also includes the analysis of plasma amino acids, urine organic acids, and plasma carnitine (free and total). Depending on results of these studies, additional testing may also be warranted.

\section{Disorders biochemically detectable by acylcarnitine analysis}

ACP analysis detects disorders of FAO and some disorders of organic acid metabolism. Specific disorders detected in a typical acylcarnitine run are shown in Table 1.

\section{Prevalence}

The combined incidence of these disorders is between $1: 5,000$ and $1: 10,000$ live births. ${ }^{12}$ 
Table 1

Inborn errors of metabolism detected by acylcarnitine profile analysis: disorders of fatty acid oxidation and organic acid metabolism

Fatty acid oxidation disorders

Carnitine uptake defect

Carnitine palmitoyltransferase I (CPTI) deficiency

Carnitine-acylcarnitine translocase (CACT) deficiency

Carnitine palmitoyltransferase II (CPTII) deficiency

Very long-chain acyl-CoA dehydrogenase (VLCAD) deficiency

Long-chain L-3-hydroxyacyl-CoA dehydrogenase (LCHAD) deficiency

Trifunctional protein (TFP) deficiency

Medium-chain acyl-CoA dehydrogenase (MCAD) deficiency

Medium/short-chain L-3-hydroxyacyl-CoA (M/SCHAD) dehydrogenase deficiency

Medium-chain 3-ketoacyl-CoA thiolase (MCKAT) deficiency

Short-chain acyl-CoA dehydrogenase (SCAD) deficiency

Multiple-CoA dehydrogenase (MAD) deficiency ( $\alpha$-ETF, $\beta$-ETF, ETF-QO deficiency) (Glutaric acidemia type II)

Dienoyl-CoA reductase deficiency

Organic acid disorders

Propionyl-CoA carboxylase deficiency (Propionic acidemia)

Multiple carboxylase deficiency (Holocarboxylase synthetase deficiency and Biotinidase deficiency)

Methylmalonyl-CoA mutase deficiency (Methylmalonic acidemia)

Disorders of cobalamin metabolism ( $\mathrm{Cbl} \mathrm{A} / \mathrm{B} / \mathrm{C} / \mathrm{D} / \mathrm{F}$ deficiencies $)$

Succinyl-CoA synthetase deficiency (SUCLA2)

Isobutyryl-CoA dehydrogenase deficiency

Ethylmalonic encephalopathy

$\beta$-Ketothiolase (2-methylacetoacetyl-CoA thiolase, or 3-oxothiolase) deficiency

Isovaleryl-CoA dehydrogenase deficiency (Isovaleric acidemia)

2-Methylbutyryl-CoA dehydrogenase deficiency (short/branched chain acyl-CoA dehydrogenase (SBCAD) deficiency)

2-Methyl 3-hydroxybutyryl-CoA dehydrogenase (MHBD) deficiency

3-Methylcrotonyl-CoA carboxylase (3-MCC) deficiency

3-Hydroxy-3-methylglutaryl-CoA lyase (HMG-CoA lyase) deficiency

3-Methylglutaconyl-CoA hydratase deficiency

Malonyl-CoA carboxylase deficiency

Glutaryl-CoA dehydrogenase deficiency (Glutaric acidemia type I)

Glutamate formiminotransferase deficiency (Formiminoglutamic aciduria)

\section{Mode of inheritance}

All IEM detectable by acylcarnitine analysis are inherited as autosomal recessive traits except 2-methyl-3-hydroxyburyrylCoA dehydrogenase deficiency, which is X-linked. ${ }^{13}$

\section{PREANALYTICAL REQUIREMENTS}

\section{Sample types}

Carnitine and its esters are found in virtually all biological fluids. The preferred sample type for the testing of symptomatic patients and asymptomatic at-risk individuals is plasma or serum. Some laboratories also prefer testing dried blood spots, an approach that facilitates ease of specimen transport and may increase the sensitivity of detecting disorders of longchain FAO. ACP is less frequently performed in urine, although this can be a sensitive approach for detecting certain organic acidemias such as glutaric acidemia type $\mathrm{I},{ }^{14}$ biotinidase deficiency, and isobutyryl-CoA dehydrogenase deficiency. ${ }^{15}$ ACP can also be performed in various tissues including cultured fibroblasts, lymphocytes, and amniocytes, as well as homogenized liver and muscle. ${ }^{16-18}$ Postmortem screening can be performed on dried-blood spots or bile, ${ }^{19,20}$ and amniotic fluid is used for the prenatal diagnosis of selected IEM. ${ }^{21}$

\section{Sample volumes}

Sample collection volumes and conditions of sample shipping and handling must be established by the laboratory and made available to referring physicians upon request. Typical sample testing volumes are given in Table 2.

\section{Conditions of sample shipping, handling and storage}

Specimens spotted and dried on filter paper can be shipped at room temperature. All other sample types should be frozen as soon as possible and shipped to the testing laboratory on dry ice. Long-term storage at room temperature causes progressive losses of acylcarnitines, with degradation of short-chain species occurring faster than long chain. Compounds are stable indefinitely when stored at $-80^{\circ} \mathrm{C}$. Criteria for sample rejection should be established and documented by the laboratory.

\section{METHOD VALIDATION}

\section{Calibration and quantitation}

Stable-isotope internal standards are available from a variety of commercial sources. These standards must be validated by

Table 2

Typical sample testing volumes for acylcarnitine profile analysis

\begin{tabular}{|c|c|}
\hline Sample type & Testing volume \\
\hline Amniotic fluid (cell-free supernatant) & $20 \mu \mathrm{L}$ \\
\hline Amniocyte culture medium & $50 \mu \mathrm{L}^{a}$ \\
\hline Bile (liquid specimen) & $20 \mu \mathrm{L}^{b}$ \\
\hline Bile dried spot (postmortem) & one $1 / 8^{\prime \prime}$ punch $^{b}$ \\
\hline $\begin{array}{l}\text { Blood dried spot (neonatal, } \\
\text { postmortem) }\end{array}$ & one $1 / 8$ " or $3 / 16$ " punch \\
\hline Cell culture medium & $50 \mu \mathrm{L}^{a}$ \\
\hline Plasma/serum & $20 \mu \mathrm{L}$ \\
\hline Urine & Variable; standardized to \\
\hline
\end{tabular}

${ }^{a}$ Spotted on filter paper.

${ }^{b}$ May require dilution if profile is abnormal. 
determining recoveries from weighed, nonisotopic acylcarnitine standards for as many compounds as possible. Internal standards must be periodically revalidated at a frequency determined by the laboratory.

Reference standards are not available for all acylcarnitine species, particularly the hydroxy species. Quantitation of these compounds should be extrapolated by application of the calibration for the nearest species of similar structure.

The laboratory should determine the linear range, analytical measurement range and lower limit of detection for all clinically informative acylcarnitines, when possible. These performance characteristics should be verified on a regular basis as specified by CLIA.

\section{Reference ranges}

Reference ranges for all reported acylcarnitines should be established and periodically validated. ${ }^{1,2}$ When literaturebased ranges are used, they must be validated by the laboratory before being put into use. For all applications except postmortem screening and tissue analyses, reference ranges should be age-matched. In particular, the comparison of pediatric results to blood spot, plasma, and serum normative ranges derived from an adult population is not appropriate.

\section{Testing personnel}

Appropriate training and ongoing competency requirements for laboratory personnel performing ACP analysis must be established and documented. Particularly for MS/MS-based applications, instrument optimization, method validation, and clinical testing must be performed by personnel with specialized training in the operation of MS/MS.

\section{ANALYSIS OF ACYLCARNITINES}

\section{Sample preparation}

Acylcarnitines are most often analyzed as their butyl-esters, ${ }^{2}$ although the analysis of underivatized specimens is possible and alternative derivatization methods (including methylation) are also occasionally used. A typical procedure for plasma sample preparation for acylcarnitine analysis is summarized below (specific procedures must be determined and validated by each laboratory):

Specimens are diluted with a mixture of deuterium-labeled internal standards dissolved in acetonitrile acidified with $0.3 \%$ formic acid or methanol. Internal standards typically include $\mathrm{d}_{3}-\mathrm{C} 2, \mathrm{~d}_{3}-\mathrm{C} 3, \mathrm{~d}_{3}-\mathrm{C} 4, \mathrm{~d}_{9}-\mathrm{C} 5, \mathrm{~d}_{3}-\mathrm{C} 8, \mathrm{~d}_{9}-\mathrm{C} 14, \mathrm{~d}_{3}-\mathrm{C} 16$. Other internal standards can also be used, but care must be taken not to interfere with the signal of a clinically relevant species (e.g., the butyl ester of $\mathrm{d}_{3}-\mathrm{C} 6$ at $m / z 319$ is only $1 \mathrm{Da}$ from $m / z 318$, the $[\mathrm{M}+1]^{+}$molecular ion of hydroxyisovalerylcarnitine). Samples are centrifuged, and the supernatant containing the extracted acylcarnitines is transferred to another tube and evaporated to dryness under nitrogen. Acylcarnitines are derivatized to their butyl-esters by adding $3 \mathrm{~N} \mathrm{HCl}$ in $n$-butanol and heating at $65^{\circ} \mathrm{C}$ for 15 minutes. Samples are again evaporated to dryness under nitrogen, and finally reconstituted in the mobile phase.

\section{Analytical methods}

For MS/MS-based testing, acylcarnitines are analyzed without chromatographic separation in the positive-ion mode, using either precursor-ion (also called parent-ion) scan or multiple-reaction monitoring (MRM). Precursor ion scan is preferable, as it allows evaluation of the whole profile and detection of common drug artifacts, presence of interfering compounds, and evidence of poor derivatization. Chromatographic separation can be considered when the separation of isomers is desired and clinically relevant. However, this is more easily achieved by other testing avenues such as urine organic acid or acylglycine analysis.

The liquid sample is introduced into the ion source through a capillary tube and exposed to a strong electric field and a counter flow of nitrogen gas (electrospray ionization, ESI). This causes evaporation of the solvent and desorption of charged ions into the mass analyzer. ESI occurs under atmospheric pressure allowing efficient ionization, while the mass analyzers operate at very low pressure. The combination is possible by introducing the ions into the analyzers through a very small $(<1 \mathrm{~mm})$ orifice and using powerful vacuum pumps.

In a standard triple quadrupole system, the first $\left(\mathrm{Q}_{1}\right)$ and third $\left(\mathrm{Q}_{3}\right)$ quadrupoles can be set either to scan a mass range or to select one or more individual ions. The second quadrupole $\left(Q_{2}\right)$ is utilized as a collision cell where fragmentation of ions in transit is enhanced. In the precursor-ion mode for the analysis of butylated derivatives, all precursors of products $\mathrm{m} / \mathrm{z} 85$ within a mass range between 200 and $500 \mathrm{~m} / z$ are recorded. For MRM analysis, appropriate parent-product ion pairs must be identified for the unambiguous detection of each reported acylcarnitine species. However, this may not always be possible. For example, the parent-product ion pair of C4-acylcarnitine $(288 / 85)$ can represent butyrylcarnitine, isobutyrylcarnitine, and formiminoglutamate. ${ }^{22}$

Acylcarnitine species are listed in Table 3, along with the molecular weight of $[\mathrm{M}+\mathrm{H}]^{+}$ions corresponding to their butyl-ester derivatives. Tolerance limits for the identification of acylcarnitines by molecular mass must be defined by the laboratory.

\section{Quality control}

A positive and negative control sample should be prepared and analyzed with every batch of patients tested. The internal standard mix should also be analyzed separately with each batch.

A quality control (QC) program based on the quantitative analysis of normal and abnormal control specimens should be implemented on a regular basis. Target ranges should be established for each metabolite and used to accept or reject a given QC run. The internal standards in each specimen serve as a QC check for each specimen. The use of Westgard rules for clinical specimen analysis further controls the parameters for quality patient diagnosis and reporting. ${ }^{23} \mathrm{~A}$ QC designee should per- 
Table 3

$[\mathrm{M}+\mathrm{H}]^{+}$ions of acylcarnitine-butyl-esters

\begin{tabular}{|c|c|c|}
\hline Acyl-carnitine (butyl-esters) & Chain length & {$[\mathrm{M}+\mathrm{H}]^{+}$} \\
\hline Free carnitine & - & 218 \\
\hline Acetyl- & $\mathrm{C} 2$ & 260 \\
\hline Acrylyl- & C3:1 & 272 \\
\hline Propionyl- & C3 & 274 \\
\hline Formiminoglutamate (FIGLU) & - & 287 \\
\hline Isobutyryl-/butyryl- & $\mathrm{C} 4$ & 288 \\
\hline Tiglyl- & C5:1 & 300 \\
\hline Isovaleryl-/2-methylbutyryl- $\left({ }^{*}\right)$ & C5 & 302 \\
\hline 3-hydroxybutyryl- & $\mathrm{C} 4-\mathrm{OH}$ & 304 \\
\hline Hexanoyl- & C6 & 316 \\
\hline $\begin{array}{l}\text { 3-hydroxyisovaleryl- 2-methyl-3- } \\
\text { hydroxybutyryl- }\end{array}$ & $\mathrm{C} 5-\mathrm{OH}$ & 318 \\
\hline Benzoyl- & - & 322 \\
\hline Dextrose (fragment) & - & 325 \\
\hline Heptanoyl- & $\mathrm{C} 7$ & 330 \\
\hline 3-hydroxyhexanoyl- & $\mathrm{C} 6-\mathrm{OH}$ & 332 \\
\hline Phenylacetyl- & - & 336 \\
\hline Salicylyl- $\left(^{*}\right)$ & - & 338 \\
\hline Octenoyl- $\left(^{*}\right)$ & C8:1 & 342 \\
\hline Octanoyl- & $\mathrm{C} 8$ & 344 \\
\hline Malonyl- & C3-DC & 360 \\
\hline Decadienoyl- & $\mathrm{C} 10: 2$ & 368 \\
\hline Decenoyl- & $\mathrm{C} 10: 1$ & 370 \\
\hline Decanoyl- & $\mathrm{C} 10$ & 372 \\
\hline Methylmalonyl-/succinyl- & C4-DC & 374 \\
\hline 3-hydroxydecenoyl- & $\mathrm{C} 10: 1-\mathrm{OH}$ & 386 \\
\hline Glutaryl-/3-hydroxydecanoyl- & $\mathrm{C} 5-\mathrm{DC} / \mathrm{C} 10-\mathrm{OH}$ & 388 \\
\hline Dodecenoyl- & $\mathrm{C} 12: 1$ & 398 \\
\hline Dextrose (fragment) & - & 399 \\
\hline Dodecanoyl- & $\mathrm{C} 12$ & 400 \\
\hline 3-hydroxydodecenoyl- & $\mathrm{C} 12: 1-\mathrm{OH}$ & 414 \\
\hline 3-hydroxydodecanoyl- & $\mathrm{C} 12-\mathrm{OH}$ & 416 \\
\hline Tetradecadienoyl- & $\mathrm{C} 14: 2$ & 424 \\
\hline Tetradecenoyl- & C14:1 & 426 \\
\hline Tetradecanoyl- (myristoyl-) & $\mathrm{C} 14$ & 428 \\
\hline 3-hydroxytetradecenoyl- & $\mathrm{C} 14: 1-\mathrm{OH}$ & 442 \\
\hline 3-hydroxytetradecenoyl- & $\mathrm{C} 14-\mathrm{OH}$ & 444 \\
\hline Hexadecenoyl- & C16:1 & 454 \\
\hline Hexadecanoyl- (palmitoyl-) & $\mathrm{C} 16$ & 456 \\
\hline 3-hydroxyhexadecenoyl- ${ }^{a}$ & $\mathrm{C} 16: 1-\mathrm{OH}$ & 470 \\
\hline \multirow[t]{2}{*}{ 3-hydroxyhexadecanoyl- } & $\mathrm{C} 16-\mathrm{OH}$ & 472 \\
\hline & & (Continued) \\
\hline
\end{tabular}

Table 3

Continued

\begin{tabular}{llc}
\hline Acyl-carnitine (butyl-esters) & Chain length & {$[\mathrm{M}+\mathrm{H}]^{+}$} \\
\hline Dextrose (fragment) & - & 473 \\
Octadecadienoyl- & $\mathrm{C} 18: 2$ & 480 \\
Octadecenoyl- & $\mathrm{C} 18: 1$ & 482 \\
Octadecanoyl- (stearyl-) & $\mathrm{C} 18$ & 484 \\
3-hydroxyoctadecadienoyl- & $\mathrm{C} 18: 2-\mathrm{OH}$ & 496 \\
3-hydroxyoctadecenoyl- & $\mathrm{C} 18: 1-\mathrm{OH}$ & 498 \\
3-hydroxyoctadecanoyl- & $\mathrm{C} 18-\mathrm{OH}$ & 500 \\
\hline
\end{tabular}

${ }^{a}$ An apparent elevation could be related to a drug and/or plasticizer interference.

form monthly compilation and review and sign-off of control values.

\section{Proficiency testing}

An ongoing proficiency testing (PT) program for acylcarnitine analysis must be implemented and documented. A biannual PT program for ACP is offered by the College of American Pathologists (CAP) and supervised by the joint CAP/ American College of Medical Genetics Genetic Biochemical and Molecular Genetic Resource Committee. ${ }^{24}$ A qualitative blood spot acylcarnitine quality assurance program is also offered by the "European Research Network for Evaluation and Improvement of Screening, Diagnosis and Treatment of Inherited Disorders of Metabolism."25

\section{TEST INTERPRETATION AND REPORTING}

\section{Interpretation}

Results should be reviewed and interpreted by an ABMGcertified biochemical geneticist. Informative results are usually characterized by a pattern of one or more elevated acylcarnitine species in comparison with age-matched reference ranges. Interpretation of results is based on pattern recognition and correlation of positive and negative findings, rather than on individual abnormal values. The differential diagnosis of abnormal results of species potentially representing multiple isomers (C4, C5, C5-OH, C5-DC) is critical and cannot be overstated. ${ }^{2,3}$ Basic acylcarnitine patterns associated with various disease states are listed in Table 4 . In addition to the primary markers listed, calculation of ratios (for example C8/C10 ratio in MCAD deficiency) is useful in the interpretation of abnormal results. Informative profiles may not always be detected in disorders where the accumulation of diagnostic species is a reflection of the residual activity of the defective enzyme, the dietary load of carnitine and precursors, and the anabolic-catabolic status of the patient. Modest abnormalities should be correlated to the patient's carnitine levels as a status of carnitine deficiency could affect the outcome of this analysis.

Several drugs (for example, valproic acid, pivalic acid, cefotaxime) may induce abnormal levels of one or more known 
Table 4

Basic acylcarnitine patterns associated with various disease states

\begin{tabular}{|c|c|c|}
\hline Designation & Acylcarnitine name & Disorder \\
\hline \multirow[t]{2}{*}{$\mathrm{C} 0 \downarrow$} & Free carnitine & Carnitine uptake defect \\
\hline & & Secondary carnitine deficiencies (e.g., in organic acidemias and FAOs) \\
\hline $\mathrm{C} 0 \uparrow$ & Free carnitine & CPTI deficiency (with C16, C18 $\downarrow$ ) \\
\hline \multirow[t]{3}{*}{$\mathrm{C} 3 \uparrow$} & Propionyl & Propionic acidemia \\
\hline & & Methylmalonic acidemias \\
\hline & & Succinyl-CoA synthetase deficiency \\
\hline \multirow[t]{5}{*}{$\mathrm{C} 4 \uparrow$} & Butyryl & SCAD deficiency \\
\hline & & Formiminoglutamic aciduria (with more prominent peak at m/z 287) \\
\hline & & Ethylmalonic encephalopathy (with C5) \\
\hline & & Multiple acyl-CoA dehydrogenase (MAD) deficiency (with C5, and other longer chain species) \\
\hline & Isobutyryl & Isobutyryl-CoA dehydrogenase (IBD) deficiency \\
\hline \multirow[t]{2}{*}{$\mathrm{C} 5 \uparrow$} & Isovaleryl & Isovaleric acidemia \\
\hline & Methylbutyryl & Methylbutyryl-CoA dehydrogenase (SBCAD) deficiency \\
\hline $\mathrm{C} 4-\mathrm{OH} \uparrow$ & 3-Hydroxybutyryl & SCHAD deficiency \\
\hline \multirow[t]{7}{*}{$\mathrm{C} 5-\mathrm{OH} \uparrow$} & 3-Hydroxyisovaleryl & 3-Methylcrotonyl-CoA carboxylase (3-MCC) deficiency \\
\hline & & Holocarboxylase synthetase deficiency \\
\hline & & HMG-CoA lyase deficiency (with C6DC) \\
\hline & & Biotinidase deficiency \\
\hline & & 3-Methylglutaconyl-CoA hydratase deficiency \\
\hline & 3-Hydroxy-2-methylbutyryl & 3-Oxothiolase deficiency (with C5:1) \\
\hline & & 2-Methyl-3-hydroxybutyryl-CoA dehydrogenase (MHBD) deficiency \\
\hline $\mathrm{C} 8 \uparrow$ & Octanoyl & MCAD deficiency (with C6, C10, C10:1) \\
\hline $\mathrm{C} 3 \mathrm{DC} \uparrow$ & Malonyl & Malonyl-CoA carboxylase deficiency \\
\hline $\mathrm{C} 4 \mathrm{DC} \uparrow$ & succinyl/methylmalonyl & Succinyl-CoA synthetase (SUCLA2) deficiency \\
\hline $\mathrm{C} 5 \mathrm{DC} \uparrow$ & Glutaryl & Glutaric acidemia type I \\
\hline $\mathrm{C} 10-\mathrm{OH} \uparrow$ & 3-Hydroxy decanoyl & M/SCHAD deficiency, MCKAT deficiency \\
\hline $\mathrm{C} 14: 1 \uparrow$ & Tetradecenoyl & VLCAD deficiency (with C14, C14:2) \\
\hline \multirow[t]{2}{*}{$\mathrm{C} 16 \uparrow$} & Palmitoyl & CPTII deficiency (with C18:2, C18:1, C18) \\
\hline & & Carnitine/acylcarnitine translocase (CACT) deficiency (with C18:2, C18:1, C18) \\
\hline \multirow[t]{2}{*}{$\mathrm{C} 16-\mathrm{OH} \uparrow$} & 3-Hydroxypalmitoyl & LCHAD deficiency (with C16:1-OH, C18:1-OH, C18-OH) \\
\hline & & Trifunctional protein (TFP) deficiency (with C16:1-OH, C18:1-OH, C18-OH) \\
\hline
\end{tabular}

acylcarnitine species. ${ }^{26,27}$ Other exogenous compounds (drug metabolites, food additives, intravenous fluids containing dextrose) could result in the appearance of atypical species, ${ }^{28}$ some of them at $m / z$ values in close proximity or even overlapping with acylcarnitines of diagnostic significance. Dietary artifacts are also possible, usually related to food intake enriched with fatty acids (MCT-oil, ketogenic diet). ${ }^{29}$

\section{Reporting}

Patient reports must contain appropriate patient and specimen information as contained in Section C 2.4, 2.4.1, and
2.4.2 of these guidelines, and as described previously. ${ }^{30}$ When abnormal results are detected, the interpretation should include an overview of the results and their significance, quantitative results with age-appropriate reference ranges, a correlation to available clinical information, elements of differential diagnosis, recommendations for additional biochemical testing and in vitro confirmatory studies (e.g., enzyme assay, molecular analysis), and a phone number to reach the reporting laboratory with additional questions. Acylcarnitine reference values are age-dependent, requiring that quantitative results be matched against a properly defined age group. 


\section{ACKNOWLEDGMENTS}

The authors thank Dr. Larry Sweetman for his comments. The revised document was reviewed and endorsed by the Biochemical Genetics Working Group of the ACMG Quality Assurance Committee.

\section{References}

1. Rinaldo $\mathrm{P}, \mathrm{Hahn} \mathrm{SH}$, Matern D. Inborn errors of amino acid, organic acid, and fatty acid metabolism. In: Burtis CA, Ashwood ER, Bruns DE, editors. Tietz textbook of clinical chemistry and molecular diagnostics, 4th ed. Philadelphia: WB Saunders, 2005:2207-2247.

2. Matern D. Acylcarnitine analysis. In: Blau N, Duran M, Gibson KM, editors. Laboratory guide to the methods in biochemical genetics. Berlin: Springer. In press.

3. Sweetman L, Millington DS, Therrell BL, Hannon WH, et al. Naming and counting disorders (conditions) included in newborn screening panels. Pediatrics 2006;17(5 Pt 2):S308-S314.

4. Chace DH. Mass spectrometry in the clinical laboratory. Chem Rev 2001;101:445477.

5. Millington DS. Tandem mass spectrometry in clinical diagnosis. In: Blau N, Duran M, Blaskovics ME, Gibson KM, editors. Physician's guide to the laboratory diagnosis of metabolic diseases, 2nd ed. Berlin: Springer, 2003:57-75.

6. Chace DH, Kalas TA. A biochemical perspective on the use of tandem mass spectrometry for newborn screening and clinical testing. Clin Biochem 2005;38:296-309.

7. Rinaldo P, Matern D, Bennett MJ. Fatty acid oxidation disorders. Annu Rev Physiol 2002;64:16.1-26.

8. Cuthbert CD, Tortorelli S, Ensenauer R, Rinaldo P, Matern D. Inherited abnormalities in mitochondrial fatty acid oxidation. In: Walker WA, Goulet OJ, Kleinman RE, Sanderson IR, et al, editors. Walker's pediatric gastrointestinal disease, 4 th ed. Hamilton, Ontario: BC Decker, 2004:1287-1304.

9. Olpin SE. Fatty acid oxidation defects as a cause of neuromyopathic disease in infants and adults. Clin Lab 2005;51:289-306.

10. Blau N, Duran M, Blaskovics ME, Gibson KM, editors. Physician's guide to the laboratory diagnosis of metabolic diseases, 3nd ed. Berlin: Springer, 2006.

11. Rinaldo P. Organic acids. In: Blau N, Duran M, Gibson KM, editors. Laboratory guide to the methods in biochemical genetics. Berlin: Springer. In press.

12. Watson MS, Lloyd-Puryear MA, Mann MY, Rinaldo P, et al. Newborn screening: Toward a uniform screening panel and system [main report]. Genet Med 2006; 8(Suppl):12S-252S.

13. Ofman R, Ruiter JP, Feenstra M, Duran M, et al. 2-Methyl-3-hydroxybutyryl-CoA dehydrogenase deficiency is caused by mutations in the HADH2 gene. Am J Hum Genet 2003;72:1300-1307.

14. Tortorelli S, Hahn SH, Cowan TM, Brewster TG et al. The urinary excretion of glutarylcarnitine is an informative tool in the biochemical diagnosis of glutaric acidemia type I. Mol Genet Metab 2005;84:137-143.

15. Oglesbee D, He M, Majumder N, Vockley J, et al. Development of a newborn screen- ing follow-up algorithm for the diagnosis of isobutyryl-CoA dehydrogenase deficiency. Genet Med 2007;9:108-116.

16. Shen JJ, Matern D, Millington DS, Hillman S, et al. Acylcarnitines in fibroblasts of patients with long-chain 3-hydroxyacyl-CoA dehydrogenase deficiency and other fatty acid oxidation disorders. J Inherit Metab Dis 2000;23:27-44.

17. Roe DS, Yang BZ, Vianey-Saban C, Struys E, et al. Differentiation of long-chain fatty acid oxidation disorders using alternative precursors and acylcarnitine profiling in fibroblasts. Mol Genet Metab 2006;87:40-47.

18. van Vlies N, Tian L, Overmars H, Bootsma AH, et al. Characterization of carnitine and fatty acid metabolism in the long-chain acyl-CoA dehydrogenase-deficient mouse. Biochem J 2005;387:185-193.

19. Chace DH, DiPerna JC, Mitchell BL, Sgroi B, et al. Electrospray tandem mass spectrometry for analysis of acylcarnitines in dried postmortem blood specimens collected at autopsy from infants with unexplained cause of death. Clin Chem 2001;47: 1166-1182.

20. Rashed MS, Ozand PT, Bennett MJ, Barnard JJ, et al. Diagnosis of inborn errors of metabolism in sudden death cases by acylcarnitine analysis of postmortem bile. Clin Chem 1995;41:1109-1114.

21. Rinaldo P, Studinski A, Matern D. Prenatal diagnosis of disorders of fatty acid transport and mitochondrial oxidation. Prenat Diagn 2001;21:52-54.

22. Malvagia S, La Marca G, Casetta B, Gasperini S, et al. Falsely elevated C4-carnitine as expression of glutamate formiminotransferase deficiency in tandem mass spectrometry newborn screening. J Mass Spectrom 2006;41:263-265.

23. Westgard JO, Klee GG. Quality Management. In: Burtis CA, Ashwood ER, Bruns DE, editors. Tietz textbook of clinical chemistry and molecular diagnostics, 4 th ed. Philadelphia: WB Saunders, 2005:485-429.

24. CAP/ACMG Biochemical and Molecular Genetics Resource Committee. Committees and leadership. Available at: http://www.cap.org/

25. European Research Network for evaluation and improvement of screening, Diag nosis and treatment of Inherited disorders of Metabolism (ERNDIM). Available at: http://www.erndimqa.nl/

26. Abdenur JE, Chamoles NA, Guinle AE, Schenone AB et al. Diagnosis of isovaleric acidaemia by tandem mass spectrometry: false positive result due to pivaloylcarnitine in a newborn screening programme. J Inherit Metab Dis 1998;21:624-630.

27. Vianey-Saban C, Boyer S, Levrat V, Cheillan D, et al. Interference of Cefotaxime in plasma acylcarnitine profile mimicking an increase of 3-hydroxypalmitoleylcarnitine (C16:1-OH) using butyl esters. J Inherit Metab Dis 2004;27(Suppl 1):94

28. Magera MJ, Hahn S, Tortorelli S, Rinaldo P, Matern D. Dextrose-an artifact detectable by newborn screening as a butylated acylcarnitine. J Inherit Metab Dis 2005; 28(Suppl 1):1.

29. Iacobazzi V, Pasquali M, Singh R, Matern D, et al. Response to therapy in carnitine/ acylcarnitine translocase (CACT) deficiency due to a novel missense mutation. Am J Med Genet 2004;126A:150-155.

30. Grier RE, Gahl WA, Cowan T, Bernardini I, et al. Revised sections F7.5 (quantitative amino acid analysis) and F7.6 (qualitative amino acid analysis): American College of Medical Genetics Standards and Guidelines for Clin Genet Laboratories, 2003. Genet Med 2004;6:66-68.

\section{PUBLISHER'S NOTE}

Driscoll DA, Gross SJ; Professional Practice and Guidelines Committee. First trimester diagnosis and screening for fetal aneuploidy. Genet Med 2008:10(1):73-75.

Lori Oxendine was incorrectly listed as an author of the above referenced article in the January issue's Table of Contents. We regret the error. 